\title{
A Note on a Generalisation of a Method of Douglas
}

\section{By Graeme Fairweather}

1. Abstract and Introduction. In this note, the high-order correct method of Douglas [1] for the diffusion equation in one space variable is extended to $q \leqq 3$ space variables. The resulting difference equations are then solved using the A. D. I. technique of Douglas and Gunn [3]. When $q=2$, this method is equivalent to that of Mitchell and Fairweather [5] while $q=3$ provides a method which is similar to Samarskiî's method [6] and of higher accuracy than that of Douglas [2].

When the proposed methods are used to solve the diffusion equation with timeindependent boundary conditions, they have the advantage that no boundary modification (see [4]) is required to maintain accuracy.

2. Derivation of Difference Equations. Consider the initial-boundary value problem

$$
\begin{aligned}
\frac{\partial u}{\partial t} & =\sum_{i=1}^{q} \frac{\partial^{2} u}{\partial x_{i}{ }^{2}}, \quad(\mathbf{x}, t) \in R \times(0, T), \\
u(\mathbf{x}, 0) & =g(x), \quad(\mathbf{x}, t) \in R \times\{0\}, \\
u(\mathbf{x}, t) & =f(\mathbf{x}, t), \quad(\mathbf{x}, t) \in \partial R \times[0, T],
\end{aligned}
$$

where $\mathbf{x}=\left(x_{1}, \cdots, x_{q}\right) \in[0,1]^{q} \equiv I_{q}, R$ is the interior of $I_{q}$ and $\partial R$ its boundary. A set of grid points with space increments $\Delta x_{i}=1 / h,(i=1, \cdots, q)$ where $N h=1$ and time increment $\Delta t=T / M$ where $N$ and $M$ are integers is imposed on the region $\bar{R} \times[0, T]$, where $\bar{R}=R+\partial R$. Denote by $w_{n}$ an approximation to $u(\mathbf{x}, t)$ $=u_{n}$ at the grid point $\left(m_{1} h, m_{2} h, \cdots, m_{q} h, n \Delta t\right)$ where $m_{i}=0,1, \cdots, N$, $(i=1, \cdots, q)$ and $n=0,1, \cdots, M$.

To derive the high order methods, we observe that

$$
\frac{u_{n+1}-u_{n}}{\Delta t}=\left(\frac{\partial u}{\partial t}\right)_{n+1 / 2}+O\left((\Delta t)^{2}\right)
$$

and

$$
\begin{aligned}
\left(\sum_{i=1}^{q} \frac{\partial^{2} u}{\partial x_{i}{ }^{2}}\right)_{n+1 / 2}= & \frac{1}{2} \sum_{i=1}^{q} \Delta_{x_{i}}^{2}\left(u_{n+1}+u_{n}\right)-\frac{h^{2}}{12} \sum_{i=1}^{q}\left(\frac{\partial^{4} u}{\partial x_{i}{ }^{4}}\right)_{n+1 / 2} \\
& +O\left(h^{4}+(\Delta t)^{2}\right),
\end{aligned}
$$

where $\Delta_{x_{i}}^{2}=\left(1 / h^{2}\right) \delta_{x_{i}}^{2}, \delta_{x_{i}}^{2}$ being the usual central difference operator.

Now

$$
\sum_{i=1}^{q} \frac{\partial^{4} u}{\partial x_{i}{ }^{4}}=\left(\sum_{i=1}^{q} \frac{\partial^{2}}{\partial x_{i}{ }^{2}}\right)^{2} u-2 \sum_{i=1 ;>>i}^{q-1} \frac{\partial^{4} u}{\partial x_{i}{ }^{2} \partial x_{j}{ }^{2}} .
$$

Thus

Received May 27, 1968. 


$$
\begin{aligned}
\left(\sum_{i=1}^{q} \frac{\partial^{4} u}{\partial x_{i}{ }^{4}}\right)_{n+1 / 2}= & \frac{1}{\Delta t} \sum_{i=1}^{q} \Delta_{x_{i}}^{2}\left(u_{n+1}-u_{n}\right)-2 \sum_{i=1 ; j>i}^{q-1} \Delta_{x_{i}}^{2} \Delta_{x_{j}}^{2} u_{n} \\
& +O\left(h^{2}+(\Delta t)^{2}+h^{4} / \Delta t\right) .
\end{aligned}
$$

Hence if we set $r=\Delta t / h^{2}$, the scheme

$$
\begin{aligned}
\frac{w_{n+1}-w_{n}}{\Delta t}= & \frac{1}{2} \sum_{i=1}^{q} \Delta_{x_{i}}^{2}\left(w_{n+1}+w_{n}\right)-\frac{1}{12 r} \sum_{i=1}^{q} \Delta_{x_{i}}^{2}\left(w_{n+1}-w_{n}\right) \\
& +\frac{\Delta t}{6 r} \sum_{i=1 ; j>i}^{q-1} \Delta_{x_{i}}^{2} \Delta_{x_{j}}^{2} w_{n}
\end{aligned}
$$

is locally fourth-order correct in space and second-order correct in time.

By an analysis similar to that presented in [2] it can be shown that if $q \leqq 3$ the solution of the difference equation (2.2) converges in the mesh $L_{2}$ norm on $R$, the global discretisation error being fourth-order correct in space and second-order correct in time.

3. A. D. I. Technique. The use of (2.2) in practice would require the solution of a large system of linear equations at each time step. This problem may be simplified by the use of the Douglas-Gunn A. D. I. technique [3]. Equation (2.2) may be rewritten in the form

$$
\begin{aligned}
{\left[1-\frac{\Delta t}{2}\left(1-\frac{1}{6 r}\right) \sum_{i=1}^{q} \Delta_{x_{i}}^{2}\right] w_{n+1}-[1} & +\frac{\Delta t}{2}\left(1+\frac{1}{6 r}\right) \sum_{i=1}^{q} \Delta_{x_{i}}^{2} \\
& \left.+\frac{1}{6 r}(\Delta t)^{2} \sum_{i=1 ; j>i}^{q-1} \Delta_{x_{i}}^{2} \Delta_{x_{j}}^{2}\right] w_{n}=0
\end{aligned}
$$

which can be solved by constructing a sequence $\beta_{n+1}^{(1)}, \cdots, \beta_{n+1}^{(q-1)}, \beta_{n+1}^{(q)} \equiv w_{n+1}$ of intermediate solutions in the following way:

$$
\begin{aligned}
& {\left[1-\frac{\Delta t}{2}\left(1-\frac{1}{6 r}\right) \Delta_{x_{1}}^{2}\right] \beta_{n+1}^{(1)}} \\
& =\left[1+\frac{\Delta t}{2}\left(1+\frac{1}{6 r}\right) \Delta_{x_{1}}^{2}+\Delta t \sum_{i=2}^{q} \Delta_{x_{i}}^{2}+\frac{(\Delta t)^{2}}{6 r} \sum_{i=1 ; j>i}^{q-1} \Delta_{x_{i}}^{2} \Delta_{x_{j}}^{2}\right] w_{n} \\
& {\left[1-\frac{\Delta t}{2}\left(1-\frac{1}{6 r}\right) \Delta_{x i}^{2}\right] \beta_{n+1}^{(i)}=\beta_{n+1}^{(i-1)}-\frac{\Delta t}{2}\left(1-\frac{1}{6 r}\right) \Delta_{x_{i}}^{2} w_{n},} \\
& i=2, \cdots, q .
\end{aligned}
$$

Thus the intermediate solutions are obtained by solving only tridiagonal systems of equations.

It is interesting to note that if $q=2$ and the intermediate solution $\beta_{n+1}^{()}$is eliminated from (3.2) we obtain the formula derived in [5]. In a similar way, the formula presented by Samarskiu [6] is obtained from (3.2) when $q=3$.

4. Stability and Accuracy of A. D. I. Method. The stability of (3.2) is proved by modifying Theorem 2.2 of [3]. This modification is necessary since the operators

$$
A_{i} \equiv\left\{-\frac{\Delta t}{2}\left(1-\frac{1}{6 r}\right) \Delta_{x_{i}}^{2}\right\}
$$


are not positive semidefinite for all values of $r$.

THEOREM. Let (3.1) be written in the form

$$
(I+A) w_{n+1}+B w_{n}=0
$$

where $A=\sum_{i=1}^{q} A_{i}, A_{i}$ given by (4.1). Since the difference operators $A_{1}, \cdots, A_{q}, A$ and $B$ satisfy

1. $I / q+A_{i}$ is positive definite, $i=1, \cdots, q$,

2. $B$ is Hermitian,

3. $A_{1}, \cdots, A_{q}, B$ commute,

the stability of (3.1) implies the stability of (3.2).

The proof follows the same lines as that of Douglas and Gunn if we make use of

Lemma. If $I / q+A_{i}$ is positive definite, $i=1, \cdots, q$, then $\sum_{2 \leqq|\sigma| \leqq q} A_{\sigma}$ is positive semidefinite where $\sigma=\left(i_{1}, i_{2}, \cdots, i_{m}\right), i_{1}<i_{2}<\cdots<i_{m},|\sigma|=m$ and $A_{\sigma}=A_{i_{1}} A_{i_{2}} \cdots A_{i_{m}}$.

That (3.2) is globally fourth-order correct in space and second-order correct in time is an immediate consequence of Theorem 2.3 of [3].

5. Intermediate Boundary Values. If the boundary conditions are time dependent the boundary conditions for the intermediate solutions $\beta_{n+1}^{(i)}(i=1, \cdots, q-1)$ appearing in (3.2) must be chosen in a particular way in order that the global error of (3.2) remain $O\left(h^{4}+(\Delta t)^{2}\right)$ otherwise a loss of accuracy will occur. For example, if $q=2$ and the boundary values at the intermediate step are chosen to be those at the time level $(n+1) \Delta t$, it can be shown that the global error is then $O\left((\Delta t)^{2} / h^{3 / 2}\right.$ $\left.+h^{4}+(\Delta t)^{2}\right)$ and if the boundary values at time level $(n+1 / 2) \Delta t$ are chosen an even worse error results. To maintain the $O\left(h^{4}+(\Delta t)^{2}\right)$ accuracy the boundary values at the intermediate step should be determined from the second of formulae (3.2) in the manner prescribed in [4]. If $q=3$, accuracy can be maintained by carrying out a similar procedure.

It can also be shown using the techniques developed in [4] that no boundary modification is required at the intermediate levels when the boundary conditions are independent of time. In particular, formulae (3.2) with $q=2$ can be used as an iterative method for solving Laplace's equation in two space variables without any cumbersome boundary modification like that required by the method proposed in [4]. The new procedure will provide more accurate approximations than the Peaceman-Rachford method [3] with little additional computational effort.

University of St. Andrews

Fife, Scotland

1. J. Douglas, JR., "The solution of the diffusion equation by a high order correct difference equation,"J. Math. Phys., v. 35, 1956, pp. 145-151. MR 19, 884.

2. J. Douglas, JR., "Alternating direction methods for three space variables," Numer. Math., v. 4, 1962, pp. 41-63. MR 24 \#B2122.

3. J. Douglas, JR. \& J. E. GunN, "A general formulation of alternating direction methods. I. Parabolic and hyperbolic problems," Numer. Math., v. 6, 1964, pp. 428-453. MR 31 \#894.

4. G. Fairweather \& A. R. Mitchell, "A new computational procedure for A.D.I. methods," SIAM J. Numer. Anal., v. 4, 1967, pp. 163-170. MR 36 \#1116.

5. A. R. Mitchell \& G. FAIRWEATHER, "Improved forms of the alternating direction methods of Douglas, Peaceman and Rachford for solving parabolic and elliptic equations," Numer. Math., v. 6,1964 , pp. $285-292$. MR $30 \# 4391$.

6. A. A. SAMARSkIY, "A difference scheme for increasing the order of accuracy for the heat equation in several variables," $\ddot{Z}$. Vyčisl. Mat. $i$ Mat. Fiz., v. 4, 1964, pp. 161-165. MR 31 \#4197. 